\title{
Study on Mechanical behavior and equivalent calculation of GFRP- stainless Steel Composite Truss Bridge
}

\author{
Hao Xianwu ${ }^{1 *}$, Liu Yuan ${ }^{1}$ \\ ${ }^{1}$ Xi'an 710000, School of Highway, Chang'an University)
}

\begin{abstract}
GFRP is a kind of carbon fiber composite reinforced material, which has attracted wide attention in the engineering field because of its light weight, high strength, corrosion resistance and so on. The solid plate and shell finite element model of full GFRP truss bridge is established by ABAQUS, and its mechanical characteristics are analyzed. The study shows that the stress of each element is less than the material strength, but the mid-span deflection of the truss bridge exceeds the allowable value of the specification, so three kinds of stainless steel plate layout schemes are considered, and the structural gravity, mid-span deflection change and element stress are compared and analyzed. Finally, it is concluded that the upper and lower stainless steel plate has a great contribution to enhance the vertical stiffness of the GFRP truss bridge. The stainless steel plate largely replaces the upper chord under pressure and the lower chord is pulled. On this basis, the stainless steel plate beam filled between oblique bars is equivalent to the upper and lower chords by using the idea of average equivalence and the principle of equal mass. The finite element software analysis shows that the equivalent error is very small.
\end{abstract}

\section{Introduction}

Bridge engineering has been exposed to the open air for a long time, there are serious structural durability problems in reinforced concrete bridge structures, in which steel corrosion and fatigue are very prominent ${ }^{[1,2]}$. In the United States, the roof system of extruded profile truss made by MBCS Company has successfully solved the corrosion problem of light steel roof truss ${ }^{[3]}$. GFRP truss bridge is a new type of bridge structure that replaces traditional truss bridge members with members made of carbon fiber composite reinforced materials. Compared with traditional steel or concrete materials, GFRP has better properties of light weight, high strength, corrosion resistance and fatigue resistance, and can meet the requirements of bridge engineering durability and construction speed. GFRP profile belongs to anisotropic material. The high fiber tensile strength of GFRP material can be brought into full play in the axial stress members of truss bridges, and its compressive strength can generally meet the requirements. Therefore, truss bridges are widely used in composite materials, which are mainly used in footbridges with small spans and projects with rapid erection requirements ${ }^{[4]}$. The University of Petre in Greece has developed a bridge made of GFRP extruded hollow square tubes ${ }^{[5]}$. The dead weight is only 13.5 tons, which can meet the vehicle load of 30 tons. Mao Yisheng Public Welfare Bridge is the first truss bridge with fiber reinforced composites in China. Feng Peng of Tsinghua University and Tian $\mathrm{Ye}^{[6]}$ used finite element software to analyze its static and dynamic performances. the results show that the load efficiency of the bridge is high. and the structure has a strong safety reserve. If the GFRP profile is used in the whole bridge, the vertical stiffness of the structure is small because of the low elastic modulus of the GFRP material, and the deflection in the middle of the span becomes the main consideration in the design. This paper analyzes the stress and deformation of the GFRPstainless steel plate beam composite truss bridge by arranging the stainless steel plate beam between the internodes, and makes a theoretical analysis of the equivalent calculation of the stainless steel plate beam.

\section{Study on Mechanical Properties of GFRP Laminates.}

FRP material can be made into fiber reinforced material products by pultrusion process. The product has good properties, high strength and low density, but it is essentially a kind of composite material, which is quite different from the physical and mechanical properties of traditional materials, especially in the relationship between stress and strain. The structural form of this kind of laminate, as shown in figure 1 , should be studied by the theory of anisotropic elasticity ${ }^{[7]}$.

*Communication author:Hao Xianwu cldbxlw@163.com 


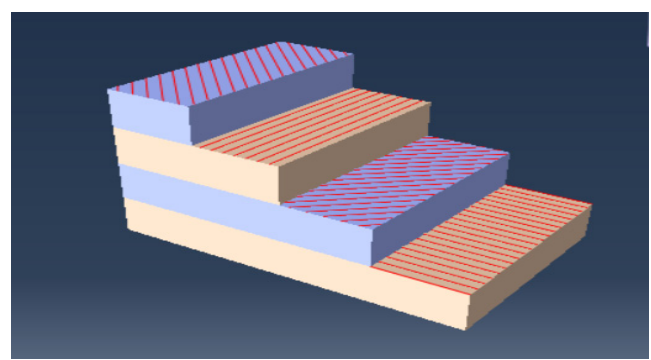

Figure 1. structural form of laminate
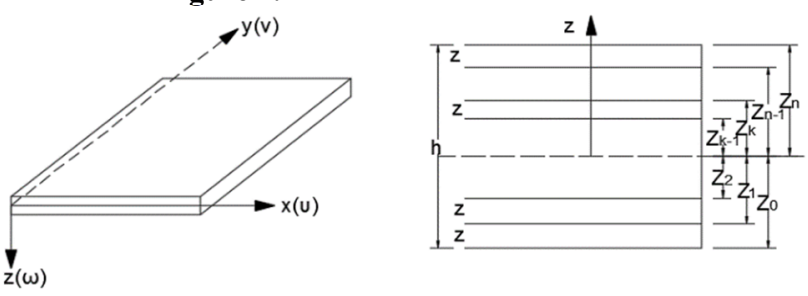

Figure 2. coordinates of laminate and delamination

The laminate can be regarded as a material composed of multi-layer single-layer laminates, and the basic mechanical model is shown in figure 2.The classical laminate theory makes the following assumptions: (1) the interlaminar deformation is consistent and there is no relative displacement. (2) the assumption that the straight normal is invariant. (3) each single layer is in a plane stress state. (4) the normal stress in the thickness direction of the laminate is ignored. The stress-strain relationship of the $\mathrm{k}$ layer of the laminate is as follows:

$$
\left[\begin{array}{c}
\sigma_{x} \\
\sigma_{y} \\
\tau_{x y}
\end{array}\right]_{k}=\left[\begin{array}{lll}
\overline{Q_{11}} & \overline{Q_{12}} & \overline{Q_{16}} \\
\overline{Q_{16}} & \overline{Q_{26}} & \overline{Q_{26}}
\end{array}\right]\left\{\left[\begin{array}{c}
\varepsilon^{0}{ }_{x} \\
\varepsilon^{0}{ }_{y} \\
\gamma_{x y}^{0}
\end{array}\right]+z\left[\begin{array}{c}
-\frac{\partial^{2} w}{\partial x^{2}} \\
-\frac{\partial^{2} w}{\partial y^{2}} \\
-2 \frac{\partial^{2} w}{\partial x \partial y}
\end{array}\right]\right\}
$$

$\varepsilon^{0}{ }_{\mathrm{x}}, \varepsilon^{0}{ }_{\mathrm{y}}, \gamma_{\mathrm{xy}}^{0}$ is Midplane strain. The relationship between internal force, internal moment and strain is as follows:

$$
\begin{aligned}
& \left\{\begin{array}{l}
N_{x} \\
N_{y} \\
N_{x y}
\end{array}\right\}=\left[\begin{array}{lll}
A_{11} & A_{12} & A_{16} \\
A_{21} & A_{22} & A_{26} \\
A_{16} & A_{26} & A_{66}
\end{array}\right]\left\{\begin{array}{l}
\varepsilon^{0}{ }_{x} \\
\varepsilon^{0}{ }_{y} \\
\gamma^{0}{ }_{x y}
\end{array}\right\}+\left[\begin{array}{ccc}
B_{11} & B_{12} & B_{16} \\
B_{21} & B_{22} & B_{26} \\
B_{16} & B_{26} & B_{66}
\end{array}\right]\left\{\begin{array}{l}
K_{x} \\
K_{y} \\
K_{x y}
\end{array}\right\} \\
& \left\{\begin{array}{l}
M_{x} \\
M_{y} \\
M_{x y}
\end{array}\right\}=\left[\begin{array}{lll}
B_{11} & B_{12} & B_{16} \\
B_{21} & B_{22} & B_{26} \\
B_{16} & B_{26} & B_{66}
\end{array}\right]\left\{\begin{array}{c}
\varepsilon^{0}{ }_{x} \\
\varepsilon^{0}{ }_{y} \\
\gamma^{0}{ }_{x y}
\end{array}\right\}+\left[\begin{array}{ccc}
D_{11} & D_{12} & D_{16} \\
D_{21} & D_{22} & D_{26} \\
D_{16} & D_{26} & D_{66}
\end{array}\right]\left\{\begin{array}{l}
K_{x} \\
K_{y} \\
K_{x y}
\end{array}\right\} \\
& K_{x}=-\frac{\partial^{2} w}{\partial x^{2}}, \quad K_{y}=-\frac{\partial^{2} w}{\partial y^{2}} \text { called curvature.; } \\
& K_{x y}=-2 \frac{\partial^{2} w}{\partial x \partial x} \text { called torsion rate; } \\
& A_{i j}=\sum_{K=1}^{N}\left(\overline{Q_{i j}}\right)_{k}\left(Z_{k}-Z_{k-1}\right) \text { called In-plane stiffness } \\
& D_{i j}=\frac{1}{3} \sum_{K=1}^{N}\left(\overline{Q_{i j}}\right)_{k}\left(Z_{k-1}^{3}-Z_{k-1}^{3}\right) \quad \text { called } \quad \text { bending } \\
& B_{i j}=\frac{1}{2} \sum_{K=1}^{N}\left(\overline{Q_{i j}}\right)_{k}\left(Z^{2}{ }_{k}-Z^{2}{ }_{k-1}\right) \quad \text { called coupling }
\end{aligned}
$$

\section{Analysis of Mechanical Properties of GFRP- stainless Steel Composite Truss Bridge}

Table1. GFRP material parameter table

\begin{tabular}{ccccccccc}
\hline E1 (Mpa) & E2 (Mpa) & E2 (Mpa) & Nu12 & Nu12 & Nu12 & G12 (Mpa) & G13 (Mpa) & G23 (Mpa) \\
\hline 35000 & 14600 & 14600 & 0.22 & 0.11 & 0.11 & 4840 & 4840 & 2420 \\
\hline
\end{tabular}

The calculated span of GFRP truss bridge is $40.0 \mathrm{~m}$, the height of truss is $4 \mathrm{~m}$, the chord spacing of truss is $3.98 \mathrm{~m}$, and the length of internodes is $4 \mathrm{~m}$. The cross-sectional dimensions of the members are shown in Table 2. The finite element software ABAQUS is used to analyze the whole bridge. In order to simulate the characteristics of laminates, the members are built with shell elements, and the supports are hinged on one side and sliding on the other. Although the pultrusion process is more mature, GFRP profiles of various sections can be formed at one time. But in practical engineering, it is still necessary to connect each component through the nodal plate, and the nodal plate has to bear the tension and pressure transmitted by the members in all directions. Because the material strength of GFRP material is low in the vertical fiber direction, GFRP material is not suitable to be used as the 
nodal plate of complex stress, but stainless steel plate is selected as the nodal plate of each nodal plate, the connection method is mechanical connection, and the fastener is bolted. The corresponding finite element geometric model is shown in figure 3 .

Table2. Section parameter table of components

\begin{tabular}{lcccc}
\hline Position & Section type & Height $(\mathrm{mm})$ & Width $(\mathrm{mm})$ & Web thickness $(\mathrm{mm})$ \\
\hline Chord, web, upper horizontal link & Square tube & 300 & 300 & 15 \\
Lower horizontal connection & I-beam & 300 & 200 & 15 \\
\hline
\end{tabular}

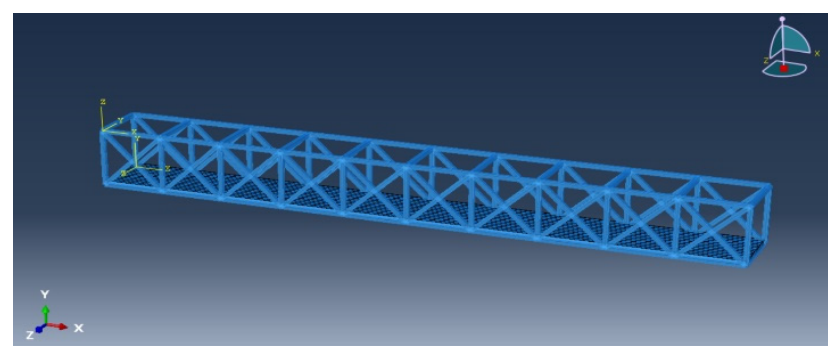

Figure 3. finite element geometric model diagram

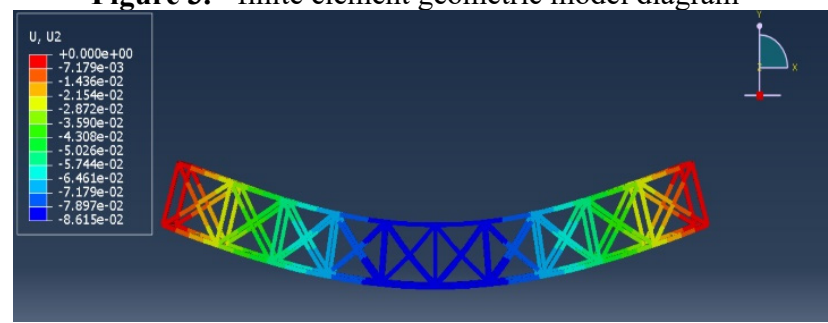

Figure 4. displacement cloud map of full GFRP truss bridge

First of all, the structural response of the whole bridge under the condition of foundation load, that is, dead weight and $5 \mathrm{kN} / \mathrm{m}^{2}$ load, is carried out. The displacement change diagram is shown in figure 4. According to the calculation results of the whole bridge model of GFRP, the maximum deflection occurs in the middle of the span, and the maximum deflection is $86.15 \mathrm{~mm}$, which is larger than the allowable value of truss bridge deflection L/800 $(50 \mathrm{~mm})$. This is due to the low elastic modulus of GFRP

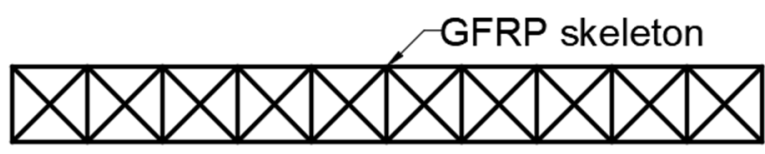
full GFRP

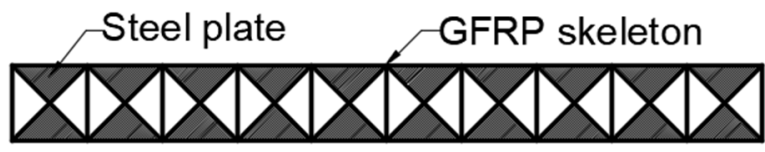

Scheme $b$ material, which leads to the small vertical stiffness of the structure, which is the main reason for limiting the span of GFRP bridge structure. On this basis, we consider the scheme of setting stainless steel beams between different oblique web members to improve the vertical stiffness of the structure. Reduce the vertical deflection in the middle of the span. The elastic modulus of stainless steel is 195GPA, Poisson's ratio is 0.247 , and the density is $7.9 \mathrm{~g} /$ $\mathrm{cm} 2$. The thickness of steel plate is $1 \mathrm{~cm}$. The shape of the steel plate beam is shown in figure 5 . The joints between the stainless steel plate and the stainless steel skeleton are mechanically connected in the form of one-sided lap connection, the fasteners are bolted, and the layout scheme is shown in figure 6 . The specific values of supporting force and mid-span deflection in four cases are shown in Table 3.

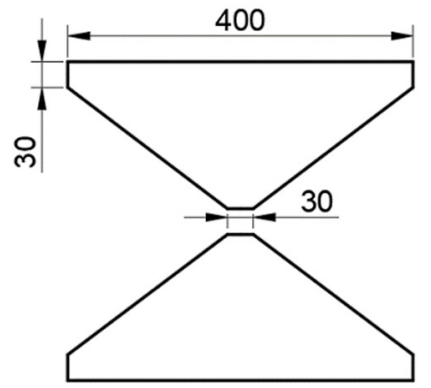

Figure 5. stainless steel plate beam diagram (unit: $\mathrm{cm}$ )

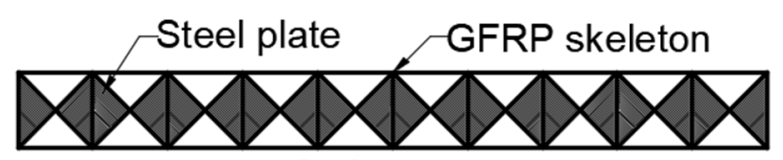

Scheme a

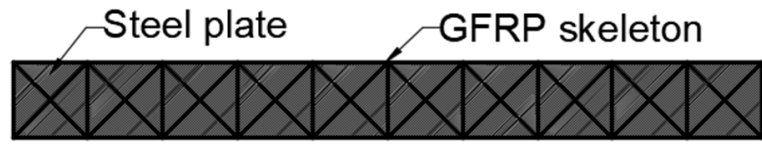

Scheme c

Figure 6. layout diagram of stainless steel plate beam

Table3. numerical table of lower support reaction and mid-span deflection of four schemes

\begin{tabular}{ccc}
\hline Scheme & Supporting force $(\mathrm{kN})$ & Mid-span deflection $(\mathrm{mm})$ \\
\hline Full FRP & 1325.936 & 86.15 \\
$\mathrm{a}$ & 1578.736 & 74.73 \\
$\mathrm{~b}$ & 1578.736 & 18.17 \\
$\mathrm{c}$ & 1831.536 & 18.04 \\
\hline
\end{tabular}


Through the data, we can draw the following conclusions: because of its material characteristics and low elastic modulus, the deformation of the full FRP truss bridge is larger than that of the steel structure, and the midspan deflection is too large, which exceeds the allowable value of the code. After setting the loaded steel plate between the inclined bars of the FRP truss bridge, the vertical stiffness of the structure changes greatly, and different steel plate layout forms have obvious influence on the results. The contrast scheme a and scheme $b$ have the same supporting force and more than 4 times difference in mid-span deflection. Through the analysis, it can be concluded that scheme $b$, which arranges triangular steel plates up and down, limits the strain of the upper and lower chords in each node, instead of the upper chord under compression. At the same time, the vertical stiffness of the whole structure is improved. Compared with scheme $\mathrm{b}$ and scheme $\mathrm{c}$, due to the arrangement of stainless steel plates on the left and right sides to increase the dead weight of the whole bridge, the reduction of midspan deflection of scheme $b$ is not significant compared with that of stainless steel plates only in the upper and lower test. Compared with the full FRP truss bridge, the gravity of scheme $b$ is increased by $252.8 \mathrm{KN}$, and the midspan deflection is reduced by $78.91 \%$. The effect is considerable. Therefore, scheme $\mathrm{b}$ is a more reasonable arrangement.By comparing the stress of the full FRP and scheme $b$, the maximum element stress of the two models appears in the middle of the span, the maximum element stress of the full FRP truss bridge is $46.23 \mathrm{MPa}$, and the maximum element stress of scheme $b$ is $7.47 \mathrm{MPa}$. through the analysis, the strain of the upper and lower chords in scheme $b$ is limited, and the stainless steel plate is used instead of FRP material, and the effect is more obvious. And the maximum element stress is far less than the strength of the material $(540 \mathrm{MPa})$, the safety reserve of the bridge is very high, and the safety is guaranteed.

\section{Equivalent calculation of GFRP- stainless steel plate beam composite truss bridge}

Considering that the structural form of GFRP- stainless steel composite truss bridge is complex, which is not convenient for design and practical engineering calculation, a simplified equivalent calculation model is given here. The basic assumptions are as follows: (1) the single laminates in GFRP laminates have reliable bonding, common force and no relative displacement. (2) the connection between GFRP material and stainless steel plate is stable at the joint. (3) the assumption of the plane section of the beam element under stress. From the analysis results of the previous chapter, it can be seen that the stainless steel plate beam plays an important role in limiting the strain of the upper and lower chords and replacing the GFRP chords after the truss bridge is subjected to stress. Moreover, the elastic modulus of stainless steel material is about 5.5times of that of GFRP material, and the mass of stainless steel material is about 4.5 times that of GFRP material. The main idea of equivalence is that the variable cross-section stainless steel plate beam filled between diagonal braces is equivalent to the equal cross-section upper and lower chord of stainless steel material. The equivalent front space variable cross-section beam is shown in figure 7 , and the coordinate origin is the upper midpoint of the left end section of the beam element. The equivalent cross section of the equal section beam is shown in 8 .

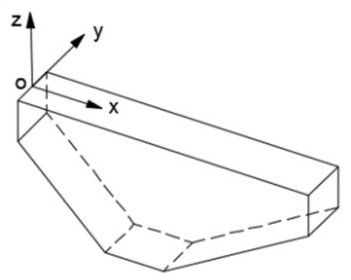

Figure 7. spatial variable cross-section beam

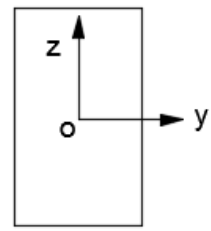

Figure 8. cross-section of equal cross-section beam

Liu Yue ${ }^{[11]}$ adopts the equivalent method that the variable cross-section beam is equivalent to the equivalent cross-section beam, and a similar equivalent idea is adopted in this chapter. In figure 7, the moments of inertia of the variable cross-section beam to the $\mathrm{x}, \mathrm{y}$ and $\mathrm{z}$ axes are:

$$
\left[\begin{array}{c}
I_{x x} \\
I_{y y} \\
I_{z z}
\end{array}\right]=\left[\begin{array}{c}
\rho \int\left(y^{2}+z^{2}\right) d v \\
\rho \int\left(z^{2}+x^{2}\right) d v \\
\rho \int\left(x^{2}+y^{2}\right) d v
\end{array}\right]
$$

$\rho$ is the bulk density of the material, the following equations can be obtained by transformation:

$$
\left[\begin{array}{l}
\int y^{2} d v \\
\int z^{2} d v
\end{array}\right]=\left[\begin{array}{c}
\frac{I_{x x}-I_{y y}+I_{z z}}{2 \rho} \\
\frac{I_{x x}+I_{y y}-I_{z z}}{2 \rho}
\end{array}\right]
$$

In the process that the variable cross-section beam is equivalent to the equivalent cross-section beam, the idea of average equivalence is put forward, that is, the concept of average quadratic moment is used to make the integration of the quadratic moments of the two crosssectional faces $o x y$ and $o x z$ equal on the $x$-axis, and the following equations are obtained:

$$
\left[\begin{array}{l}
\int_{0}^{L}\left(\int y^{2} d A\right) d x \\
\int_{0}^{L}\left(\int z^{2} d A\right) d x
\end{array}\right]=\left[\begin{array}{l}
L I_{z} \\
L I_{y}
\end{array}\right]
$$

$L$ represents the length of the beam element, A represents the cross-sectional area, and the left end of formula (8) and (9) are equal, so the following can be obtained: 


$$
\left[\begin{array}{l}
I_{z} \\
I_{y}
\end{array}\right]=\left[\begin{array}{l}
\frac{I_{x x}-I_{y y}+I_{z z}}{2 L \rho} \\
\frac{I_{x x}+I_{y y}-I_{z z}}{2 L \rho}
\end{array}\right]
$$

By the same token, the concept of equal mass is put forward, that is, the mass of the equivalent front and rear beam elements should be equal, so there are:

$$
\rho \int_{0}^{L}\left(\int d A\right) d x=\rho V=A L
$$

Simultaneous forms (10) and (11) are available:

$$
\left[\begin{array}{c}
I_{y} \\
I_{z} \\
A
\end{array}\right]=\left[\begin{array}{c}
\frac{I_{x x}+I_{y y}-I_{z z}}{2 L \rho} \\
\frac{I_{x x}-I_{y y}+I_{z z}}{2 L \rho} \\
\frac{V}{L}
\end{array}\right]
$$

The left of formula $I_{y} 、 I_{z} 、 A$ represents three unknowns of the equivalent rear beam element, and the right $I_{x x} 、 I_{y y}, I_{z z} \quad V$ can be calculated by the quality feature function of SolidWorks 3D drawing software. The equivalent section parameter $I_{y} 、 I_{z} 、 A$ of the beam element obtained by formula (12) is the same as that of the original variable cross-section beam. The equivalent equal cross-section beam element is used to replace the original GFRP main chord, and the equivalent result is verified by the finite element software. The equivalent mid-span deflection is $18.17 \mathrm{~mm}$ before the equivalent load, and the equivalent mid-span deflection is $18.95 \mathrm{~mm}$, with an error of $4.3 \%$. The error may come from two aspects. (1) for the error of variable cross-section equivalent to equal crosssection, (2) the equivalent structure ignores the bending stiffness of the GFRP truss main chord before equivalent. However, the error is small, which indicates that this equivalent method is more reasonable in calculating the maximum deflection in the span.

\section{Conclusion}

The main results are as follows: 1) under the foundation load, the maximum element stress of the main chord of the full GFRP truss bridge is $46.23 \mathrm{MPa}$, which appears in the middle section of the span, which is much smaller than the material strength, and its safety reserve is very high, with the maximum deflection up to $86.15 \mathrm{~mm}$, which is larger than the allowable value of truss bridge deflection L/800 $(50 \mathrm{~mm})$. This is due to the low elastic modulus of GFRP material, which leads to the small vertical stiffness of the structure, which is the main reason for limiting the span of GFRP bridge structure. It is the main consideration in the analysis and design of GFRP truss bridge.

2) through the comparative analysis of three kinds of stainless steel plate layout schemes, scheme b, which arranges triangular steel plates up and down, limits the strain of the upper and lower chords in each internode, replaces the tension of the upper chord under compression, and improves the vertical stiffness of the whole structure. Compared with the full FRP truss bridge, the gravity increases by $252.8 \mathrm{kN}$ and the mid-span deflection decreases by $78.91 \%$. Therefore, scheme $b$ is a more reasonable arrangement. In the case of scheme $b$, the maximum unit stress of the GFRP main chord appears in the mid-span section, which is $7.47 \mathrm{MPa}$, which indicates that the stainless steel plate bears most of the tension and pressure instead of the GFRP chord.

3 ) according to the equal mass principle, the filled stainless steel plate is equivalent to a stainless steel chord, ignoring the contribution of the mass and bending stiffness of the GFRP chord to the whole bridge structure. finally, through the finite element software simulation, taking the mid-span deflection before and after the equivalent load as the judgment condition, the equivalent mid-span deflection is $18.17 \mathrm{~mm}$, the equivalent mid-span deflection is $18.95 \mathrm{~mm}$, and the error is $4.3 \%$. It shows that this equivalent method is reasonable in calculating the maximum deflection in the middle of the span, and provides an idea for the simplified calculation of GFRPstainless steel plate truss bridge.

\section{Reference}

1. Chen Zhaoyuan, Xu Youlin, Qian Jiaru. Safety and durability of civil structure engineering [J]. Building Technology, 2002 (04): 248-253. (in Chinese)

2. Di Xiaotan, Gao Xiaowang, Xu Youlin. Durability and safety of concrete building structures in China [J]. China Construction Industry Press, 2003.1-6. (in Chinese )

3. Jane Gilby. Pultrusion provides roof solution[J]. Reinforced Plastic, 1998, 42(6):48,51-49,52.

4. Xu Yiyang, Fang Hai, Liu Weiqing. Application and Development of Composite Extruded profile in Truss Bridge structure [J]. Bridges of the World, 2010 (04): 32-34+38 (in Chinese)

5. Kostopoulos,Markopoulos,Vlachos,Katerelos, Galioti s,Tsiknias,Zacharopoulos,Karalekas,Chronis,Kaloma llos. Design and construction of a vehicular bridge made of glass/polyester pultruded box beams[J]. Plastics, Rubber and Composites, 2005, 34(4).

6. Feng Peng, Tian Ye, Qin Zhaoping. Study on static and dynamic properties of fiber reinforced composite extruded profile truss bridge [J]. Industrial Architecture, ,2013,43(06):36-41. (in Chinese)

7. Shen Guanlin, $\mathrm{Hu}$ more open. Mechanics of composite materials [M]. Tsinghua University Press, 2013. (in Chinese)

8. GB/T 1447-2005, Test method for tensile properties of fiber reinforced plastics [S]. (in Chinese)

9. GB/T 1448-2005, Test method for compressive properties of fiber reinforced plastics [S]. 
10. Chen Sipeng. Mechanical property Analysis and Experimental Research of GFRP Truss Bridge [D]. Southeast University, 2017 (in Chinese)

11. Liu Yue, Wang Liping. Simplification of spatially variable cross-section beams based on stiffness matrix [J]. Journal of Tsinghua University (Natural Science Edition), 2008 (11): 1915-1918 (in Chinese) 